\title{
A comparison of length-matching and length-fractionation measures of Müller-Lyer distortions
}

\author{
JOHN PREDEBON \\ University of Sydney, New South Wales, Australia
}

\begin{abstract}
Two experiments were performed to evaluate the informational equivalence of length-matching (e.g., reproduction) and length-fractionation (e.g., bisection) procedures that are frequently used to quantify geometrical size distortions, such as the Müller-Lyer (M-L) illusion. In Experiment 1, the distortion in the apparent length of a horizontal test line was measured as a function of the angle between it and an abutting inducing line, and in Experiment 2 distortion was measured in the apparent length of the shaft of one-angle versions of the M-L illusion. Both procedures indexed the expansion of the obtuse-angle and the wings-out M-L illusion and the contraction of the acute-angle and the wings-in M-L illusion. However, whereas the reproduction measures indicated substantially greater expansion than contraction distortion, the bisection measures indicated greater contraction distortion. Some possible reasons for this difference, particularly the possibility that the reproduction and bisection procedures are unequally sensitive to the outputs of the mechanisms producing the M-L illusion, are discussed.
\end{abstract}

Studies on visual geometric illusions try to minimize the interaction between the measurement device or task used to quantify illusion magnitude and the illusory effect being measured by ensuring that the task does not influence, and is not influenced by, the illusory effect itself. The literature, however, has ignored another kind of measurement "interaction"-namely, a procedure's differential sensitivity to the outputs of illusion-inducing mechanisms. The neglect of this issue is especially surprising, as mechanism-measurement interactions are potentially indicative of the kinds of mechanism generating a given illusory effect.

The present investigation considers the mechanismmeasurement issue with respect to two widely used procedures - the length-matching and length-fractionation methods - with reference to the well-known Müller-Lyer (M-L) illusion (see Figure 1). These procedures differ in task orientation - specifically, in whether the task directs attention to the overall length of a single test extent or to the relative lengths of segments of the test extent. Matching procedures, such as the method of reproduction, typically require judgments of the length of the test extent relative to the length of a "comparison" extent. In contrast, fractionation procedures, such as the method of bisection, typically partition a test extent into two or more apparently equal segments.

Fractionation and matching judgments of a length illusion are unlikely to yield equivalent measurement out-

Correspondence relating to this article may be sent to J. Predebon, School of Psychology, University of Sydney, Sydney, NSW 2006, Australia (e-mail: johnp@psych.usyd.edu.au). comes if, as in the case of the M-L illusion, the illusioninducing mechanisms produce dissimilar distortion gradients along the test extent. The M-L illusion is widely regarded as the resultant effect of a multiplicity of mechanisms operating at different levels of visual processing (e.g., Restle \& Decker, 1977). The outputs of some mechanisms, such as position encoding (Morgan, Hole, \& Glennerster, 1990), optical blur (Chiang, 1968), and interactions involving contour and orientation feature analyzers (Walker, 1973), produce gradients of distortions extending from the vertices, with diminishing influence along the shaft. Other mechanisms, such as size or spatial-frequency encoding processes (Ginsburg, 1984; Oyama, 1977) and length-attribute averaging processes conceived at the level of either feature extraction (e.g., Brigell, Uhlarik, \& Goldhorn, 1977) or judgment (Pressey \& Pressey, 1992), generate uniform distortions along the shaft's entire length.

The fractionation and matching procedures are unequally sensitive to uniform and nonuniform distortions; whereas matching procedures are sensitive to-can detect - both types of distortions, fractionation procedures are insensitive- "blind"- - to uniform distortions along the target extent and are sensitive only to nonuniform effects. The two procedures therefore measure different illusory effects, and examples of these differences are given in Figure 1. Figures 1A and 1B, respectively, show the wings-in and wings-out M-L illusion forms, each with a mark dividing the shaft. Despite the apparent length inequality of the two shafts, the mark appears to bisect each shaft into two equally long segments (which it does). In this instance, the bisection judgment fails to index the distortion that is evident with the comparative length judgment. Figures 1C and 1D, respectively, show 
A

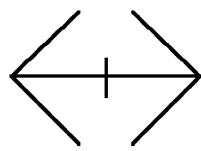

C

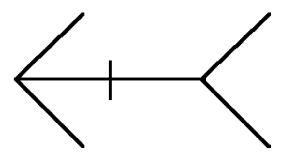

$\mathrm{E}$

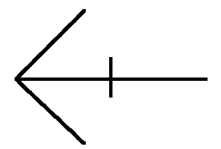

$\mathrm{B}$

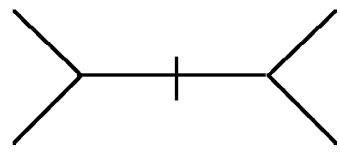

$\mathrm{D}$

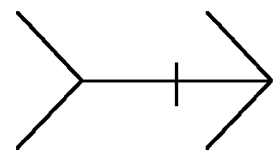

F

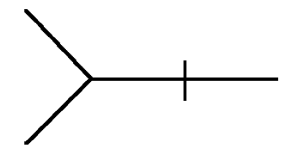

Figure 1. The wings-in or underestimated (A) and wings-out or overestimated (B) M-L illusion forms, the Judd illusion with left(C) and right- (D) pointing arrowheads, and the one-angle versions of the wings-in (E) and wings-out (F) M-L illusion. All figures are shown with a vertical bar bisecting the shaft into segments that are equally long physically.

the leftward- and rightward-pointing forms of the Judd illusion, again each with a mark dividing the shaft. Despite the apparent length equality of the two shafts, the mark appears to bisect each shaft into two unequal segments (which it does not). In this instance, the bisection judgment indexes a distortion that plainly is not captured by the comparative length judgment. Figures $1 \mathrm{E}$ and $1 \mathrm{~F}$, respectively, show the one-angle version of the classic wings-in and wings-out M-L figures, each with a dividing mark, or alternatively the one-angle version of the leftward- and rightward-pointing Judd figures. For these two figures, the shafts appear different in length (which they are not) and the dividing marks appear to bisect the shafts into unequal segments (which they do not). In this case, a distortion of the shaft is indexed by both the bisection and comparative length judgments.

The different visual effects of dividing the shafts of the M-L and Judd figures into physically equal segments are consistent with the operation in both figures of nonuniform illusory effects originating at the vertices and diminishing toward the center of the shaft (Predebon, 2001). For the M-L illusion forms (Figures 1A and $1 \mathrm{~B}$ ), the illusory effects either cancel each other out or are absent in the center of the shaft, with the result that the dividing mark is perceived correctly to be at the shaft's center. For the Judd illusion figures (1C and 1D), the effects of the end wings operate in concert, shortening the portion of the shaft adjacent to the outwardpointing wing $(->,<-)$ and lengthening the portion adjacent to the inward-pointing wing $(-<,>-)$. The net effect of this asymmetrical distortion is to shift the shaft's apparent midpoint toward the inward-pointing arrowhead, with the result that the dividing mark appears displaced toward the outward-pointing arrowhead. Finally, and for the same reason, the dividing mark in the one-angle figures $(1 \mathrm{E}$ and $1 \mathrm{~F})$ appears displaced toward the angle end of the wings-in figure and toward the open end of the wings-out figure. The effect is less than for the Judd figures, since only one angle (and therefore only one gradient of distortion) is present.

In principle, fractionating an extent into two equal segments - referred to as bisection-can only "detect" spatial distortions that are nonuniformly and asymmetrically distributed along a test extent. This sensitivity to nonuniform distortions of an extent is a major reason why the bisection task is used extensively to index neurological disorders of spatial attention, such as unilateral visuospatial neglect (e.g., Mattingley, Bradshaw, \& Bradshaw, 1995; Olk, Harvey, Dow, \& Murphy, 2001). Patients with left visual-field neglect, for example, typically bisect a horizontal line too far to the right of the objective midpoint, suggesting that their perception of the left end of the line is impaired (Bisiach, Bulgarelli, Sterzi, \& Vallar, 1983). Fractionation tasks, however, can also "detect" the localized distortion components of an extent illusion providing (1) the distortions are symmetrically distributed along the extent, as is presumed to be the case for the classic M-L illusion forms (Predebon, 2001), and (2) the extent is fractionated into more than two, such as four (Warren \& Bashford, 1977) or eight (Post, Welch, \& Caulfield, 1998; Predebon, 2001), apparently equal segments. Predebon (2001), for example, using a novel scoring procedure, which yielded an unambiguous signature of the magnitude of the distortions at various loci along the shaft, found that the nonuniform distortion components in both the wings-in and wingsout $\mathrm{M}-\mathrm{L}$ forms are localized to the regions of the shaft adjacent to the vertices, thus confirming Morinaga's (1941; cited in Oyama, 1960) early observation: Morinaga divided the shafts of the two M-L forms into seven segments and reported that all segments except for those adjacent to the vertices appeared equal in length.

The putative existence of localized and uniform illusory effects in M-L displays suggests that the fractionation and matching procedures are unlikely to measure identical patterns of wings-in and wings-out illusory effects. Although systematic comparisons between the two procedures are lacking, the results of M-L studies conducted in other contexts suggest a major intriguing difference. This evidence concerns the relative magnitudes of the wings-in and wings-out M-L illusions: In general, it seems that the matching procedure, which has been used in the majority of the studies investigating the determinants of the M-L illusion, routinely measures (e.g., Beagley, 1985; Christie, 1975; Day \& Dickinson, 1976) substantially greater distortion magnitudes-frequently almost twice as much - for the wings-out than for the wings-in illusion in both their classic (Figures 1A and 1B) and one-angle forms (Figures $1 \mathrm{E}$ and $1 \mathrm{~F}$ ).

In contrast, the results of the few fractionation studies have not consistently revealed greater wings-out bisection errors, but instead either similar wings-in and wingsout or greater wings-in errors. Warren and Bashford's 
A

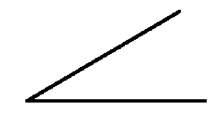

C

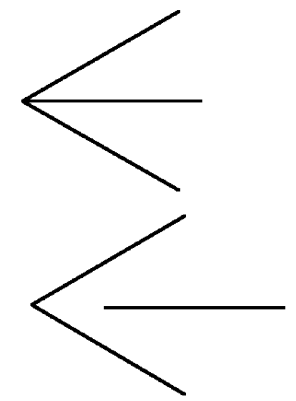

B

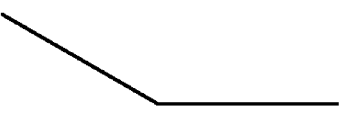

$\mathrm{D}$

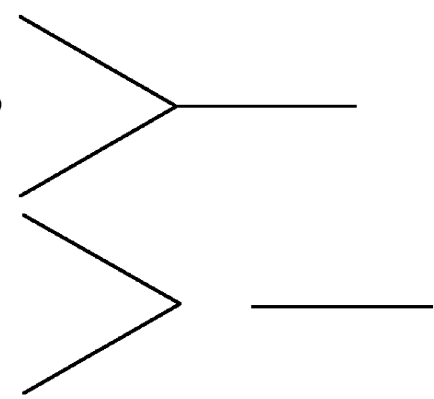

Figure 2. Examples of the stimulus figures used in Experiment 1 (A, B) and Experiment 2 (C-F).

(1977, Table 4) data for the single-fin M-L figures (see Figures 2A and 2B) indicate similar wings-out and wings-in errors. In his Experiment 3, Predebon (1994) also reported similar illusion magnitudes for the oneangle M-L figures, although his Experiment 2 data indicated slightly greater wings-out bisection errors. Predebon (2001) found slightly greater wings-in bisection errors for both the two-angle and one-angle M-L illusion figures. Likewise, Worrall and Firth (1974) measured greater errors for the one-angle wings-in illusion, and Mattingley et al. (1995) and Olk et al. (2001) found either similar or greater one-angle wings-in bisection errors.

The two procedures' different estimates of the relative magnitudes of the wings-in and wings-out M-L illusions warrant independent evaluation for at least two reasons. First, the procedures have been used in different studies, so their disparate estimates are possibly confounded with other unknown factors. Second, providing converging evidence for this difference potentially helps clarify the kinds of mechanisms responsible for the formation of the M-L illusion in the first place. The primary aim of the two experiments reported here, therefore, was to compare the results of the two procedures under conditions in which the matching and bisection judgments are made under otherwise identical stimulus and procedural conditions.

A possible complication when comparing the results of the two procedures, however, is that they may differentially recruit other perceptual and high-level cognitive processes in addition to the visual processes encoding spatial location and extent in M-L figures. For instance, work performed in other contexts (see Fischer, 2001, for a review) suggests that the bisection task can be influenced by a range of cognitive operations, depending on the orthographic, phonological, and semantic attributes of the stimulus. Macdonald-Nethercott, Kinnear, and Venneri (2000) found systematic bias in judgments of the apparent midpoint of a plain horizontal line surrounded by arrowheads all pointing in the same direc- tion - the bias was away from the direction the arrows pointed. The visual features of the end points also influence bisection, as Westheimer, Crist, Gorski, and Gilbert (2001) have demonstrated recently. They found that thresholds for detecting whether a central marker accurately bisects a spatial interval are best when the dividing marker is identical to the outer delimiters of a spatial extent, and performance is impaired by as much as a factor of two when the outer delimiters differ from the dividing marker in length, orientation, or contrast polarity. In the case of length-matching procedures, even simple contextual visual features, such as small dots placed spatially adjacent to but separated from each end of a plain line, increase a line's apparent length (Predebon, 1992). It also seems that the way spatial attention is deployed can have small but significant effects in altering the apparent lengths of lines; a briefly presented line is judged longer when it is unattended than when it is attended (Tsal \& Shalev, 1996; but see Prinzmetal \& Wilson, 1997).

In view of the (albeit unknown) possibility of the bisection and matching tasks differentially recruiting and measuring distortion components that are unrelated to the mechanisms producing the M-L illusory effect, investigating only a few one-angle $\mathrm{M}-\mathrm{L}$ figures seemed problematic. For this reason, the present experiments were designed to assess whether the effects of experimental manipulations on the magnitudes of the M-L illusion and its variants, which have already been identified and measured using length-matching procedures, are replicated using the bisection procedure. To this end, the aim of Experiment 1 was to obtain judgments of the length and apparent midpoint of the horizontal arm of simple angle figures as a function of the angle, from $0^{\circ}$ to $180^{\circ}$ (Day \& Dickinson, 1976), and the aim of Experiment 2 was to obtain judgments of the length and apparent midpoint of one-angle wings-in and wings-out M$\mathrm{L}$ forms as a function of the separation or gap between the shaft and the wing (Worrall \& Firth, 1974). 

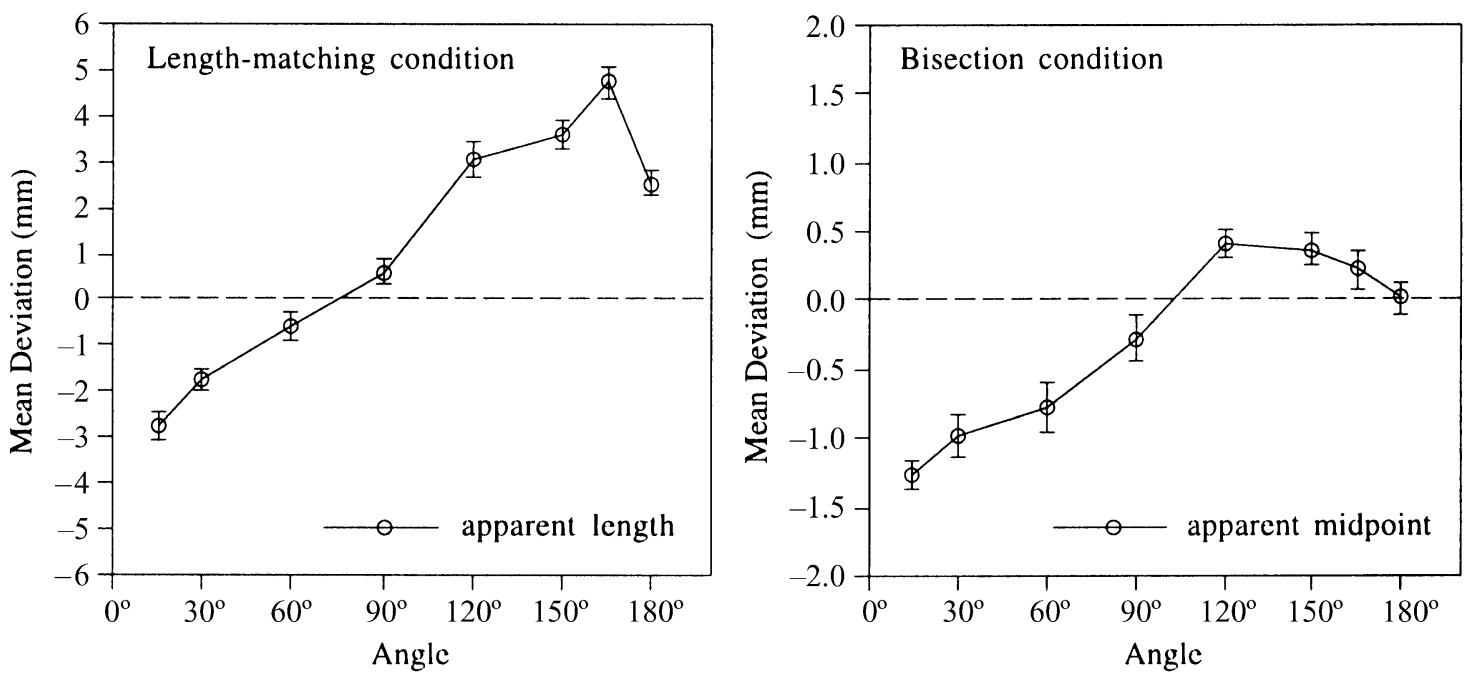

Figure 3. Experiment 1: Mean deviations of judgments of apparent length (length-matching) and apparent midpoint (bisection) from the point of subjective equality, as a function of angle. Vertical bars indicate $\pm 1 S E$.

\section{EXPERIMENT 1}

Day and Dickinson (1976) investigated a reduced form of the M-L illusion in which the angle between a horizontal test line and an attached inducing line was varied between $0^{\circ}$ and $180^{\circ}$. They found that (1) the magnitude of the length-matching errors of the test line varied systematically as a function of angle, with under- and overestimation of the line, respectively, for angles less than and greater than approximately $90^{\circ}$; and (2) the maximum overestimation of the obtuse-angle illusion exceeded the maximum underestimation of the acute-angle illusion by a factor of two. The aim of Experiment 1 was to measure the angular function of errors in judgment of the apparent midpoint of the horizontal test arm of simple angle figures. It was also necessary to include a length-matching condition in order to confirm Day and Dickinson's results for the angular function of matching errors under the stimulus and procedural conditions of this new experiment.

\section{Method}

\section{Subjects}

Twenty-three undergraduate students recruited from an introductory psychology course participated for course credit. All had normal or corrected-to-normal vision.

\section{Stimuli and Apparatus}

Each of the eight test figures contained a 50-mm-long horizontal test line and a $50-\mathrm{mm}$-long inducing line that joined the test line at its left end to form an angle of $15^{\circ}, 30^{\circ}, 60^{\circ}, 90^{\circ}, 120^{\circ}, 150^{\circ}, 165^{\circ}$, or $180^{\circ}$. Examples of the acute- and obtuse-angle stimuli are shown in Figures $2 \mathrm{~A}$ and $2 \mathrm{~B}$. The $180^{\circ}$ figure was a $100-\mathrm{mm}$ horizontal line divided by a $2-\mathrm{mm}$ cross bar. The control figure was a $50-\mathrm{mm}$ horizontal test line. The stimulus figures were presented on a $33 \times$ $25 \mathrm{~cm}$ computer monitor. All lines were one pixel wide (approximately $0.3 \mathrm{~mm}$ ) and were black against a whitish background. The displays were viewed from a distance of $57 \mathrm{~cm}$. From this distance, the $50-\mathrm{mm}$ test lines subtended a visual angle of $5^{\circ}$.
Reproduction. For the reproduction task, each test figure was presented with a variable-length comparison line. The test figure was presented in the upper left and the comparison line in the lower right quadrant of a $33 \times 25 \mathrm{~cm}$ computer monitor. The comparison and test lines were vertically separated by an average of $60 \mathrm{~mm}$. The right end of the test line and the left end of the comparison line were horizontally separated by an average of $20 \mathrm{~mm}$. On each trial, the left end of the comparison line was fixed in position, and expanding or contracting the line's right end effected changes in its length.

To prevent the subjects from utilizing fixed position reference markers in making their length matches, both the horizontal and vertical separations of the comparison and test figures were varied across successive trials by $\pm 10 \%$. The subjects adjusted the length of the comparison shaft either by moving the computer mouse or tapping the right and left arrow keys on the keyboard. Bracketing was encouraged.

Bisection. The stimuli used for the bisection condition were identical to those for the reproduction condition, except for the absence of the comparison line and the presence on the $50-\mathrm{mm}$ horizontal test line of an intersecting 2 -mm vertical marker. The subjects could adjust the marker, either by moving the mouse or tapping the right and left arrow keys of the keyboard, to any position on the test line. The figures were approximately centered on the computer screen. Once again, to prevent the subjects from using screen reference points, the precise positions of the centers of figures were randomized across successive trials within a notional $2-\mathrm{cm}$ diameter circular area centered on the screen.

\section{Procedure}

The 11 subjects in the reproduction condition were instructed to adjust the length of the comparison line so that it appeared equal to the length of the $50-\mathrm{mm}$ test line. There were two blocks of trials, each consisting of the randomized presentation of all the test figures. The subjects made two judgments of each test figure before proceeding to the next: one with the comparison line initially set noticeably longer than the test line, the other with it initially set noticeably shorter. Thus, the subjects made a total of four judgments for each stimulus figure. The subjects signaled the completion of an adjustment by pressing a key on the computer keyboard, which also caused the stimulus figure to disappear from the screen.

A different group of 12 subjects were assigned to the bisection task condition. They were instructed to position the marker so that 
it appeared to bisect the test line into two equal segments. One adjustment was made with the marker initially set so that the left segment appeared noticeably shorter than the other segment, the other with the left segment set noticeably longer. Again, bracketing was encouraged, and the two blocks of trials each consisted of the randomized presentation of the test figures. In all other respects, the procedure was identical to that for the reproduction condition.

\section{Results}

For the reproduction condition, the average of the four length reproductions of the control line provided an estimate of each subject's point of subjective equality (PSE): the overall mean of the reproductions was $49.3 \mathrm{~mm}$ $(S E=0.25)$. Each subject's length reproductions of the test lines were converted to deviations from the PSE by subtracting the subject's PSE from the length of each reproduction.

For the bisection condition, each subject's settings were scored according to the distance of the marker from the test line's physical midpoint. The subject's four scores for each figure were then averaged to produce a single score for each figure. The average of each subject's four settings on the control line provided an estimate of the PSE; the overall mean of the control-figure settings was $+0.61 \mathrm{~mm}(S E=0.16)$. The test-figure scores were converted to deviations from the PSE by subtracting the PSE from each subject's scores for the eight test lines.

The mean deviations from PSE as a function of the angle between the test and inducing lines are shown in Figure 3, separately for the reproduction and bisection judgments, as a function of the angle between the test and inducing lines. For the reproduction condition, negative and positive scores indicate under- and overestimation of the test line relative to judgments of the control line. For the bisection condition, negative and positive scores indicate that the marker was positioned farther from or closer to the vertex of the angle, respectively, relative to the position of the apparent midpoint of the control line. Negative scores therefore signify a gradient of apparent contraction of the space adjacent to the vertex - the marker had to be positioned away from the angle to compensate for the illusory length contraction. Conversely, positive scores signify a gradient of apparent expansion, resulting in a shift of the apparent midpoint toward the vertex of the angle.

As is evident from Figure 3, for both conditions the magnitude and direction of the errors are systematically dependent on stimulus angle; for angles less than $90^{\circ}$, test lines were underestimated, and for angles greater than $90^{\circ}$, they were overestimated. One-way repeated measures analyses of variance (ANOVAs) performed on the reproduction and bisection data sets separately yielded significant effects of angle for both the reproduction $[F(7,70)=80.43, p<.05]$ and bisection $[F(7,77)=$ $30.30, p<.05]$ sets.

Tests of trend indicated significant linear, quadratic, cubic, and quartic components $[F(1,10)=488.96,83.59$,
111.17 , and 13.15, respectively, $p<.05$ ] in the analysis of the reproduction data and significant linear, quadratic, and cubic components $[F(1,11)=56.02,47.21$, and 10.23 , respectively, $p<.05$ ] in the analysis of the bisection data.

Figure 3 also clearly shows an asymmetry in the magnitudes of the over- and underestimations in the lengthmatching data in the direction of substantially greater overestimation errors: The maximum overestimation error (at $165^{\circ}$ ) is significantly greater than the maximum underestimation error $\left(\right.$ at $\left.15^{\circ}\right)[t(10)=3.35, p<.05]$. An asymmetry is also evident in the bisection data, although in the opposite direction: The peak negative bisection error $\left(\right.$ at $\left.15^{\circ}\right)$ is significantly greater than the peak positive bisection error (at $\left.120^{\circ}\right)[t(11)=6.45, p<.05]$.

\section{Discussion}

The results of the reproduction condition confirm Day and Dickinson's (1976) findings and mirror the characteristics of the classic M-L illusory forms - namely, the apparent contraction and expansion of the acute-angle (or wings-in) and the obtuse-angle (or wings-out) forms, respectively, and the greater absolute magnitude of the expansion (wings-out) relative to the contraction (wingsin) illusory effect. The bisection data also show a systematic influence of angle. However, whereas the reproduction data indicate substantially greater wings-out distortions, the bisection data indicate substantially greater wings-in distortions. This difference will be discussed in detail later, in the General Discussion section.

\section{EXPERIMENT 2}

Arguably, simple angle figures may be unrepresentative of more complex illusion-inducing angle patterns, such as the classic or the one-angle M-L illusions; hence, the findings of Experiment 1 may be of limited utility. One aim of Experiment 2, therefore, was to extend the work to versions of the one-angle M-L figures shown in Figures $2 \mathrm{C}$ and $2 \mathrm{D}$. To maintain continuity with the stimulus figures of Experiment 1, the wings and shaft of the M-L figures had identical lengths.

Two bisection studies (Predebon, 1994; Worrall \& Firth, 1974) investigating the one-angle M-L forms have done so in the context of the reverse M-L effect. The reverse effect occurs when a gap is introduced between the wings and shaft of the classic M-L figures (see Figures $2 \mathrm{E}$ and $2 \mathrm{~F}$ ), and was first noted by Yanagisawa (cited in Oyama, 1960). The effect refers to the change in the relative magnitudes of the classic wings-in and wings-out illusions as a function of gap size; whereas the apparent elongation of the wings-out shaft decreases systematically, the apparent contraction of the wings-in shaft first decreases and then changes-reverses - to apparent elongation as a function of gap size. In Experiment 2, the opportunity was taken to compare, under otherwise identical stimulus and viewing conditions, the patterns of re- 
production and bisection errors for the one-angle M-L illusion forms as a function of gap size.

A reexamination of the reverse $M-L$ effect in oneangle M-L figures is warranted for two reasons. First, the results of the two extant studies are inconsistent with each other, so the robustness of the reverse effect as measured with the bisection procedure is uncertain. Worrall and Firth (1974) failed to confirm the reverse M-L effect in one-angle figures, and in fact found the exact opposite: The magnitude of wings-in bisection errors decreased but did not change direction, whereas wings-out errors reversed from positive to negative as a function of gap size. ${ }^{1}$ In contrast, Predebon (1994) confirmed the normal reverse effect, with bisection errors of the wingsin illusion reversing from negative to positive, although the magnitude of the reverse wings-in illusion was rather small: The maximum positive bisection error of the $80-\mathrm{mm}$ wings-in M-L shaft was only about $0.5 \mathrm{~mm}$.

Second, the measurement of the reverse effect with the bisection procedure implies that at least one determinant of the one-angle $\mathrm{M}-\mathrm{L}$ reverse illusion has a nonuniform effect along the shaft. However, it is difficult to identify what this nonuniform effect might be, since none of the assumed mechanisms producing nonuniform distortions predict a reversal in the direction of the illusory effect from contraction to expansion, as distinct from a reduction in the contraction effect, as a function of gap size. Confirming the measurement of the reverse M-L effect with a bisection task, therefore, would have significant implications for constraining theories of M-L distortions.

\section{Method}

\section{Subjects}

A group of 24 new subjects participated for course credit. All had normal or corrected-to-normal vision.

\section{Apparatus and Stimuli}

The test stimuli contained a 50-mm horizontal test line and two abutting oblique lines that formed an internal angle of $60^{\circ}$ on the left side of the test line. The vertex of the angle pointed horizontally either toward or away from the test line's left end. The gap between the vertex and the left end of the line varied from 0 to $20 \mathrm{~mm}$ in 5 -mm steps. This range of gaps was guided by earlier work indicating that with $50-\mathrm{mm}$ one-angle M-L figures the apparent contraction of the wings-in figure reverses to apparent elongation when the gap is between 5 and $10 \mathrm{~mm}$. There were two sets of five test figures. For one set of figures, the angle pointed to the left $(<)$, and for the other it pointed to the right $(>)$. Examples of the test figures are shown in Figures $2 \mathrm{C}-2 \mathrm{~F}$. The control stimulus was a plain $50-$ $\mathrm{mm}$ horizontal line.

For the reproduction condition, each stimulus contained a variable comparison line. The test figures were presented at the upper left of the computer monitor and the variable line at the lower left. In all other respects, the relative positions of the comparison and test lines, viewing distance, and stimulus dimensions were identical to those of the reproduction condition of Experiment 1. For the bisection condition, the M-L stimuli were similar to the figures of the reproduction condition, except for the absence of the comparison line and the presence of a $2-\mathrm{mm}$ adjustable marker intersecting the horizontal test line.

\section{Procedure}

The subjects were assigned randomly to one of the two conditions, with 12 subjects in each condition. The subjects in the reproduction condition were presented with two randomized sequences of the 10 test figures and the one control figure. In all other respects, the procedure was identical to the reproduction condition in Experiment 1. For the bisection condition, the instructions and the presentation sequence of the stimuli were identical to those of the bisection condition in Experiment 1.

\section{Results}

Data treatment was similar to that in Experiment 1. For the reproduction condition, the mean PSE of the control line was $50.3 \mathrm{~mm}(S E=0.21)$. The length judgments of the test lines were converted to deviations from the PSE by subtracting the PSE from the reproduction of each of the 10 test lines. For the bisection condition, the mean PSE of the control line was $+0.54 \mathrm{~mm}(S E=$ 0.14 ). The judgments of the test lines were converted to deviations from the PSE by subtracting the PSE from the judgments of each of the 10 test lines. Figure 4 shows the mean deviations from PSE separately for the reproduction and bisection judgments as a function of the gap between the test and inducing lines.

\section{Reproduction}

One-way repeated measures ANOVAs performed separately on the wings-in and wings-out data were significant $[F(4,44)=75.99$ and 62.66, respectively, $p<.05]$, indicating that gap size reliably influenced length reproductions. Tests of trend indicated significant linear and quadratic components for both the wings-in $[F(1,11)=$ 271.52 and 24.97, respectively, $p<.05$ ] and wings-out $[F(1,11)=241.37$ and 19.13, respectively, $p<.05]$ data sets. The cubic and quartic components were not significant for either condition.

The results confirm the occurrence of the reverse M-L effect under the conditions of the present experiment. Whereas the wings-in or underestimation illusion reversed to overestimation as a function of gap size, the wings-out or overestimation illusion decreased but did not reverse. Additionally, and in line with the results of other studies (e.g., Day \& Dickinson, 1976), for the standard (i.e., zero-gap) M-L figures the absolute magnitude of the wings-out illusion was significantly greater than that of the wings-in illusion $[t(11)=6.16, p<.05]$.

\section{Bisection}

One-way repeated measures ANOVAs performed separately on the wings-in and wings-out data sets both were significant $[F(4,44)=54.14$ and 21.17 , respectively, $p<.05]$, indicating that gap size reliably influenced judgments of a test line's apparent midpoint. A test of trend showed that the linear and quadratic components were significant in the analysis of the wings-out data $[F(1,11)=41.84$ and 10.91, respectively, $p<.05]$ as were the linear, quadratic, and cubic components in the analy- 

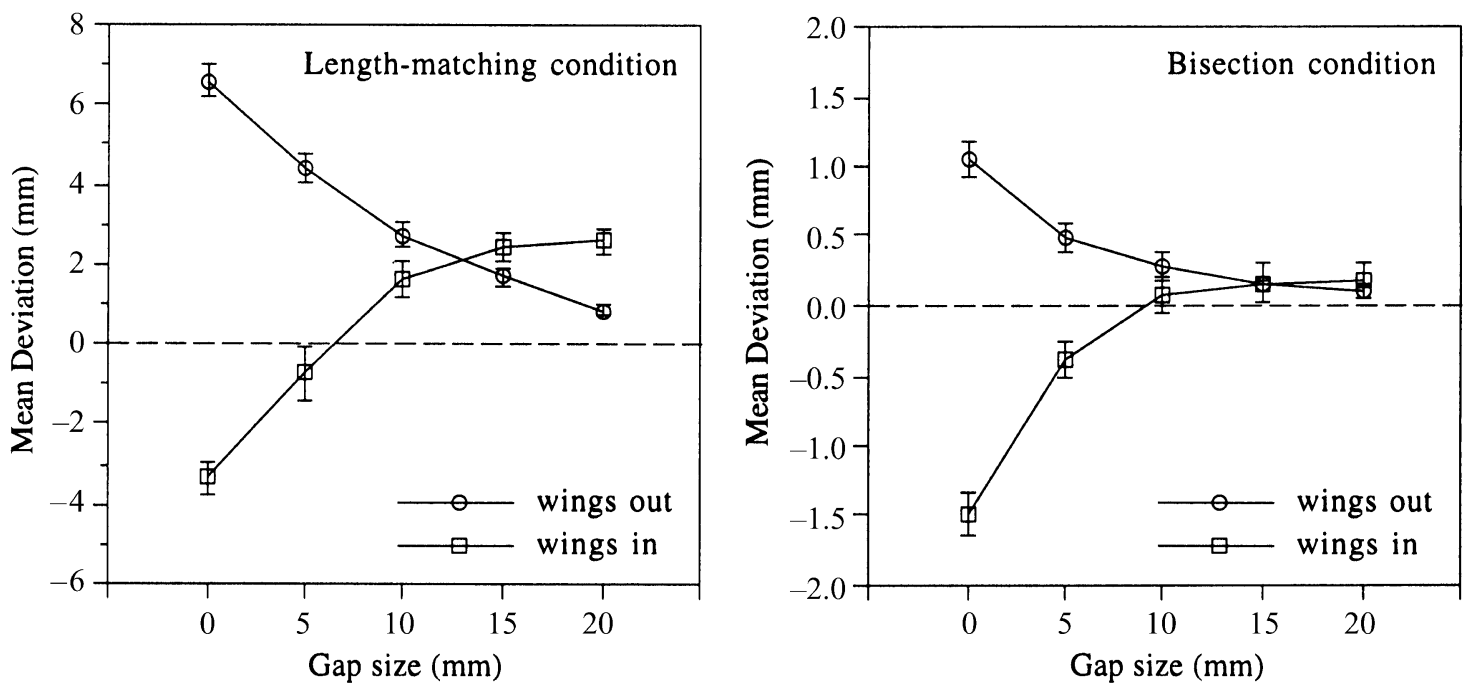

Figure 4. Experiment 2: Mean deviations of judgments of apparent length (length-matching) and apparent midpoint (bisection) from the point of subjective equality, as a function of the size of the gap between the wing vertex and the shaft for the wings-out and wings-in one-angle Müller-Lyer figures. Vertical bars indicate $\pm 1 S E$.

sis of the wings-in data $[F(1,11)=90.45,70.22$, and 6.79 , respectively, $p<.05]$. A comparison between the magnitudes of the zero-gap M-L forms was also significant $[t(11)=2.37, p<.05]$, indicating that the absolute magnitude of the bisection error was significantly greater for the wings-in than for the wings-out illusion.

\section{Discussion}

It is clear from Figure 4 that the bisection and reproduction data show similar effects of gap size on illusion magnitudes. In both cases, whereas increasing the size of the gap decreased the apparent lengthening of the wings-out shaft, as the gap increased the apparent shortening of the wings-in shaft decreased and then changed - reversed - to apparent lengthening. There are, however, two notable differences. First, the procedures yielded opposing estimates of the relative magnitudes of the standard (i.e., zero gap size) wings-in and wings-out illusory effects, with the reproduction procedure measuring a greater wings-out illusion and the bisection procedure a greater wings-in illusion. Second, the procedures differed in the relative magnitudes of the wings-in and wings-out illusory effects for the range of gap sizes (greater than about $10 \mathrm{~mm}$ ) in which the wings-in illusion reversed direction. In accord with previous findings obtained with conventional (i.e., two-angle) M-L illusion forms (Fellows, 1967; Predebon, 1992), the magnitude of the reverse wings-in illusion measured with the reproduction procedure exceeded the magnitude of the wings-out illusion, a tendency that was significant for the $20-\mathrm{mm}$ gap size condition $[t(11)=4.66, p<.05]$. For the bisection condition, however, the magnitudes of the wings-in and wingsout bisection errors were virtually identical.

\section{GENERAL DISCUSSION}

The aim of the present investigation was to determine whether or not length-matching and length-fractionation procedures for quantifying M-L illusion magnitudes, instanced respectively by the methods of reproduction and bisection, produce similar measurement outcomes. Both procedures indexed expansion for both the obtuse-angle figures and the zero-gap wings-out M-L figures and contraction for both the acute-angle figures and the zero-gap wings-in M-L figures. Additionally, both measured a reversal in the direction of the wings-in M-L distortion from underestimation to overestimation as the size of a gap between the vertex of the wings and the shaft increased. The major and novel finding of this study was a consistent difference in the measured magnitudes of the wingsin and wings-out distortions: Whereas reproduction judgments revealed substantially greater wings-out than wings-in distortions, bisection judgments indicated greater distortions for the wings-in figures.

As noted previously, the mechanisms generating M-L distortions differ in their spatial range of influence. Interestingly, most formulations of the mechanisms generating uniform distortions predict an asymmetry in wingsin and wings-out illusion magnitudes in the direction of greater wings-out illusions. For example, in both Oyama's (1977) size-tuning and Brigell et al.'s (1977) neural length-averaging models, the asymmetry in illusion magnitudes is a by-product of the interactions among neural analyzers that are distributed along a logarithmic length continuum. In contrast, the mechanisms producing localized distortions are associated with a range of relative wings-in and wings-out illusion magnitudes. Greater 
wings-in illusion magnitudes are predicted by contour interaction mechanisms in the spatial (e.g., Walker, 1973) and orientation (Carpenter \& Blakemore, 1973) ${ }^{2}$ domains, but other mechanisms, such as optical blur (Chiang, 1968) and certain formulations of position-encoding mechanisms (e.g., Morgan et al., 1990), appear to predict similar illusion magnitudes. Taken together, the mechanisms producing nonuniform distortions predict either similar wings-in and wings-out or somewhat greater wings-in illusion magnitudes.

Because a matching procedure is sensitive to both nonuniform and uniform distortions, reproduction data normally should exhibit greater wings-out than wings-in distortions. Conversely, since a bisection procedure only detects nonuniform distortion components, bisection data should show similar or slightly greater wings-in illusion magnitudes. These predictions are consistent with the results of the standard or zero-gap M-L conditions of Experiment 2: Whereas the reproduction procedure measured a wings-out illusion that was 1.98 times greater than the wings-in illusion, the bisection procedure measured a wings-in illusion that was 1.32 times greater. The results of the bisection condition of Experiment 1, however, appear inconsistent with the prediction for the bisection data. Instead of similar acute- and obtuse-angle bisection errors, the maximum negative error $\left(\right.$ at $\left.15^{\circ}\right)$ was substantially-3.2 times-greater than the maximum positive error (at $120^{\circ}$ ). This apparent discrepancy between the bisection results of Experiments 1 and 2, however, is explicable in terms of the relationships between the target extents, either the shaft of the M-L figures (Experiment 2) or the horizontal arm of the angle (Experiment 1), and the locus of the illusory effects generated by the mechanisms yielding localized M-L distortions.

The foci of the localized distortions in M-L figures and in their simple angle variants are at the vertices of the angles or wings, and their effects on perception can be described as shifting the apparent location of a vertex along the bisector of the angle components of the figures. For the M-L figures of Experiment 2, the shift occurred along the angle's bisector, which in this instance corresponded to the test extent or shaft. The shift was in the direction of the angle's interior, either toward the shaft of the wings-in figure or away from the shaft of the wings-out figure, thereby producing apparent contraction or expansion of the shaft, respectively.

For the angle figures of Experiment 1, the apparent shift of the vertex also occurred along the angle's bisector, but in this instance the bisector did not correspond to the horizontal test extent. As a result, the magnitude of the horizontal component of this shift-that is, the component producing the distortion of the horizontal test line's length - varied as a function of the angle between the induction and test lines. For acute angles, because the orientations of the angle's bisector and the test line were similar, the magnitude of the horizontal component of the shift was large, thereby producing substantial contrac- tion of the test line's length. For obtuse angles, however, the orientations of the angle's bisector (between $45^{\circ}$ and $90^{\circ}$ ) and the horizontal test line were significantly different from each other, and therefore the magnitude of the horizontal component of the shift in vertex location along the test line was rather small. Consequently, since the horizontal component of the shift is much larger for acute than for obtuse angles, the magnitude of the bisection errors should be considerably greater for the acute than for the obtuse angle figures, which is what was found in Experiment 1.

Experiment 2 confirmed the reverse M-L effect with both the matching and bisection procedures. The reverse effect measured with the matching procedure is easily explained by assimilation theories of the illusion. According to Pressey's theory (e.g., Pressey \& Bross, 1973), for example, the effect arises because the shaft assimilates to the average of the implied contextual extents formed between the free end of the shaft and the wings. When there is no gap between the shaft and the wing, all the contextual magnitudes are shorter than the shaft, with the result that the apparent length of the shaft shortens. When a small gap is introduced, some of the contextual magnitudes will be longer than the shaft. These longer contextual lines will counteract the effect of the remaining shorter lines, with the result that the illusion decreases. As gap size increases, there will come a point at which most or all of the contextual lines are longer than the shaft, thus producing lengthening - a reverse effect - rather than shortening of the shaft.

As was noted earlier, the reverse effect measured with the bisection task is not predicted by any of the mechanisms generating the nonuniform distortion components of the M-L illusion. Whereas these mechanisms produce a gradient of distortion that extends outward from the wings with diminishing influence into the adjacent space, the reverse effect implies a qualitative change of the distortion from contraction to expansion as a function of the distance from the vertex. Nor can current formulations of assimilation theory, which assume that the contextual effects operate uniformly along the entire length of the shaft, be easily modified to explain these findings. It is arguable, however, whether the reverse effects measured with the bisection and matching procedures are related. For one thing, the magnitudes of the wings-in reverse bisection errors are quite small, averaging $0.21 \mathrm{~mm}$ across the $10-, 15-$, and $20-\mathrm{mm}$ gap conditions. For another, for the range of gap sizes in which the reverse effect occurs, the magnitudes of the wings-in and wings-out bisection errors are similar, unlike the pattern of reverse $\mathrm{M}-\mathrm{L}$ reproduction errors that indicate greater wings-in than wings-out positive errors (see Figure 4).

The results of Predebon's (2001) Experiment 2, which used the fractionation method to map the pattern of spatial distortions as a function of position along the shaft, also suggest that the reverse effect measured with the bisection procedure may be unrelated to the mechanisms 
producing the M-L illusion. He had subjects partition the $100-\mathrm{mm}$ shaft of one-angle wings-in figures into eight equal segments $(<-|-|-|-|-|-|-\mid)$. The first bisection mark $(<----\mid---)$, with which subjects tried to divide the shaft into two equal segments, showed the usual illusory effect - the mark was drawn away from the shaft's midpoint, toward the free end. The judgment that fractionated the half segment of the shaft adjacent to the wing $(<--|--|----)$ also showed a significant illusory effect, as did the judgment that divided the resulting quarter segment adjacent to the wings $(<-|-|--\mid----)$. The judgment bisecting the second quarter segment from the wings $(<-|-|-|-|-\mid---)$ was performed accurately, as were the judgments bisecting the half segment of the shaft at its free end $(<----|--|--)$ and those bisecting the third $(<----|-|-\mid-)$ and fourth $(<----|-|-|-|-)$ quarters along the shaft. The pattern of these bisection results suggests that the spatial spread of the nonuniform distortions is confined to the regions adjacent to vertices, and furthermore that the contraction effect decreases but does not change to expansion, contrary to the implication of measuring a reverse effect with the bisection procedure.

Perhaps the reverse M-L bisection effect indexes a localized mechanism that is activated only when a gap is introduced between the shaft and the wing, although what this mechanism might be is difficult to speculate. The lack of parsimony, plausibility, and predictive utility of this possibility suggests that an explanation must be sought elsewhere. One possibility stems from the observation mentioned earlier that the bisection procedure might be tapping factors other than the mechanisms responsible for the encoding of position and extent in M-L figures (Fischer, 2001). Arguably, the bisection errors measured in Experiment 2 include a bisection bias component that is specific to the bisection procedure. This bias arises from the sheer presence of the angle pattern in the figure and is unrelated to the processes generating the distortions of position and extent. Its effect becomes evident in bisection judgments of one-angle M-L figures once the mechanisms producing the localized M-L illusory effects are eliminated by spatially separating the wing from the shaft, as in the reverse M-L manipulation. Since this is a general bisection bias, the magnitude and direction of the wings-in and wings-out bisection errors for the range of gap sizes for which the reverse M-L effect occurs should be similar, which is what was found in both Experiment 2 and Predebon (1994). From this perspective, then, the reverse M-L effects measured by the reproduction and bisection procedures are unrelated; the reproduction measures mainly reflect the M-L distortions of extent and position, but the bisection measures index perceptual and cognitive factors of the kind discussed by Fischer.

In summary, the present findings show that the fractionation and matching procedures do not produce identical measurement outcomes, at least with respect to measurements of the relative magnitudes of the under- and overestimated variants of the Müller-Lyer illusion. The pattern of these results is consistent with both the conjecture that M-L distortions are multiply determined, with one class of illusion-inducing mechanism producing nonuniform effects and the other yielding uniform effects along the entire M-L shaft, and the claim that the matching and fractionation procedures are unequally sensitive to the outputs of these two classes of mechanism.

\section{REFERENCES}

Beagley, W. K. (1985). Interaction of Müller-Lyer with filled-unfilled space illusion: An explanation of Müller-Lyer asymmetry. Perception \& Psychophysics, 37, 45-49.

Bisiach, E., Bulgarelli, C., Sterzi, R., \& Vallar, G. (1983). Line bisection and cognitive plasticity of unilateral spatial neglect. Brain \& Cognition, 2, 32-38.

Brigell, M., UhlariK, J., \& Goldhorn, P. (1977). Contextual influences on judgments of linear extent. Journal of Experimental Psychology: Human Perception \& Performance, 3, 105-118.

Carpenter, R. H. S., \& Blakemore, C. B. (1973). Interactions between orientations in human vision. Experimental Brain Research, 18, 287-303.

CHIANG, C. (1968). A new theory to explain geometrical illusions produced by crossing lines. Perception \& Psychophysics, 3, 174-176.

Christie, P. S. (1975). Asymmetry in the Müller-Lyer illusion: Artifact or genuine effect? Perception, 4, 453-457.

DAY, R. H., \& Dickinson, R. G. (1976). Apparent length of the arms of acute and obtuse angles, and the components of the Müller-Lyer illusion. Australian Journal of Psychology, 28, 137-148.

Fellows, B. J. (1967). Reversal of the Müller-Lyer illusion with changes in the length of the inter-fins line. Quarterly Journal of Experimental Psychology, 19, 208-214.

Fischer, M. H. (2001). Cognition in the bisection task. Trends in Cognitive Sciences, 5, 460-462.

Ginsburg, A. P. (1984). Visual form perception based on biological filtering. In L. Spillman \& B. R. Wooten (Eds.), Sensory experience, adaptation, and perception: Festschrift for Ivo Kohler (pp. 53-72). Hillsdale, NJ: Erlbaum.

Macdonald-Nethercott, E. M., Kinnear, P. R., \& Venneri, A. (2000). Effect of a directional cue on line bisection. Brain \& Cognition, 43, 325-328.

Mattingley, J. B., Bradshaw, J. L., \& Bradshaw, J. A. (1995). The effects of unilateral visuospatial neglect on perception of Müller-Lyer illusory figures. Perception, 24, 415-433.

Morgan, M. J., Hole, G. J., \& Glennerster, A. (1990). Biases and sensitivities in geometrical illusions. Vision Research, 30, 1793-1810.

OlK, B., Harvey, M., Dow, L., \& MurPhy, P. J. S. (2001). Illusion processing in hemispatial neglect. Neuropsychologia, 39, 611-625.

Oyama, T. (1960). Japanese studies on the so-called geometrical optical illusions. Psychologia, 3, 7-20.

Oyama, T. (1977). Feature analyzers, optical illusions, and figural aftereffects. Perception, 6, 401-406.

Post, R., Welch, R., \& Caulfield, K. (1998). Relative spatial expansion and contraction within the Müller-Lyer and Judd illusions. Perception, 27, 827-838.

Predebon, J. (1992). Framing effects and the reversed Müller-Lyer illusion. Perception \& Psychophysics, 52, 307-314.

Predebon, J. (1994). The reversed Müller-Lyer illusion in conventional and in wing-amputated Müller-Lyer figures. Psychological Research, 56, 217-223

PREDEBON, J. (2001). Spatial range of illusory effects in Müller-Lyer figures. Psychological Research, 65, 226-234.

Pressey, A. W., \& Bross, M. (1973). Assimilation theory and the reversed Müller-Lyer illusion. Perception, 2, 211-217.

Pressey, A. W., \& Pressey, C. A. (1992). Attentive fields are related to focal and contextual features: A study of Müller-Lyer distortions. Perception \& Psychophysics, 51, 423-436. 
PrinZmetal, W., \& Wilson, A. (1997). The effect of attention on phenomenal length. Perception, 26, 193-205.

Restle, F., \& Decker, J. (1977). Size of the Müller-Lyer illusion as a function of its dimensions: Theory and data. Perception \& Psychophysics, 21, 489-503.

Tsal, Y., \& SHALEV, L. (1996). Inattention magnifies perceived length: The attentional receptive field hypothesis. Journal of Experimental Psychology: Human Perception \& Performance, 22, 233-243.

WALKER, E. H. (1973). A mathematical theory of optical illusions and figural aftereffects. Perception \& Psychophysics, 13, 467-486.

WARREN, R. M., \& BASHFord, J. A. (1977). Müller-Lyer illusions: Their origin in processes facilitating object recognition. Perception, 6, 615626.

Westheimer, G., Crist, R. E., Gorski, L., \& Gilbert, C. D. (2001). Configuration specificity in bisection acuity. Vision Research, $\mathbf{4 1}$ 1133-1138.

WORRALL, N., \& FiRTH, D. (1974). The components of the standard and reverse Müller-Lyer illusions. Quarterly Journal of Experimental Psychology, 26, 342-354.

\section{NOTES}

1. Worrall and Firth (1974) did not reference the illusory effects to the PSE for a control line. Instead, they calculated illusion magnitudes with respect to the physical midpoint of the shaft. Conceivably, had they done their measurements with reference to the PSE, they might have obtained a pattern of results consistent with the normal reverse M-L effect (Predebon, 1994).

2. Carpenter and Blakemore's (1973) work essentially confirms the phenomenon of acute-angle expansion and obtuse-angle contraction. Given a few simplifying assumptions, including the covariation of the orientation and position domains and the pivoting of orientation shift toward the center of each of the angle's arms (i.e., away from the vertex), acute-angle expansion and obtuse-angle contraction produce apparent shifts of vertex location either toward or away from the angle's interior, respectively. Carpenter and Blakemore found that the magnitude of acute-angle expansion was greater than that of obtuse-angle contraction, a finding that is consistent with the greater bisection errors in the acute- than in the obtuse-angle bisection condition of Experiment 1.

(Manuscript received May 8, 2003;

revision accepted for publication May 17, 2004.) 